\title{
Analysis of diffusion and extraction in hollow cylinders for some boundary conditions
}

\author{
Ching Chiang Hwang ${ }^{1}$, Ing-Bang Huang ${ }^{2 *}$ \\ ${ }^{1}$ Department of Biotechnology, Mingdao University, Taiwan \\ ${ }^{2 *}$ Department of Materials Science and Engineering, National Formosa University, Huwei, Yulin, 63201,
}

Taiwan (corresponding author)

\begin{abstract}
Analysis of diffusion and extraction in hollow cylinders with different outer / inner radius ratio has been investigated. The first five roots, $\alpha_{n}$ of the two valuable equations, $J_{I}(a \alpha) Y_{0}(b \alpha)-J_{0}(b \alpha) Y_{I}(a \alpha)=0$ and $J_{0}(a \alpha) Y_{1}(b \alpha)-J_{l}(b \alpha) Y_{0}(a \alpha)=0$, were derived and tabulated. Under the condition where diffusion coefficient is constant, the concentration profiles curves of diffusion and extraction for some cases have been demonstrated and discussed.
\end{abstract}

Keywords: - Diffusion; Transport properties; Mechanical properties

\section{INTRODUCTION}

Because of a sensitive electrochemical method developed by Devanathan and Stachurski [1] and some mathematical solutions of the pertinent diffusion equation given by McBreen et al. [2], Kiuchi and McLellan [3], and Yen and Shih [4], measurements of the diffusion coefficient and the permeation rate of hydrogen through a metal membrane have been widely investigated. When critical hydrogen concentration induced cracking in a metal pipe (hollow cylinder) has become an important factor [5-6]. Therefore, the need to understand the concentration profile in a hollow cylinder might be urgent. Several decades ago, Ash et al. [7] provided a means of measuring the diffusion coefficient $\mathrm{D}$ for a material in the form of a hollow cylinder shell by the time lag method. Carslaw and Jaeger [8-9] also gave some solutions to the problem of heat conduction through a hollow cylinder shell with some initial and boundary conditions. Crank [10] applied the above mathematics to diffusion for hollow cylinder shells for some cases. However, a more general mathematical solution including steady, set up transient, and decay transient states of the concentration distribution and permeation rate in a hollow cylinder was investigated in our earlier study [11].

The objective of this study was to derive the mathematical solutions of diffusion and extraction in hollow cylinders with different outer / inner radius ratio K. In this paper, the concentration profiles for set up transient and decay transient states were given, respectively.

\subsection{Diffusion equation}

\section{MathematiCAl ANALYSIS}

Consider a long circular cylinder in which diffusion is everywhere radial. Concentration is then a function of radius $r$ and time $t$ only, and the diffusion equation becomes

$$
\frac{\partial C}{\partial t}=\frac{1}{r} \frac{\partial}{\partial r}\left(r D \frac{\partial C}{\partial r}\right)
$$

\subsection{Set up transient state}

Carslaw and Jaeger [8] have given the solution to the problem of diffusion into a hollow cylinder in which the concentration is initially zero and the boundary conditions on the two surfaces are

$$
\begin{aligned}
& k_{1} \frac{\partial C}{\partial r}-k_{2} C=k_{3}, r=a \\
& k_{1}^{\prime} \frac{\partial C}{\partial r}+k_{2}^{\prime} C=k_{3}^{\prime}, r=b
\end{aligned}
$$


Carslaw and Jaeger [8] give the solution by the use of Laplace transformation. The final result is

$C=\frac{-a k_{3}\left[k_{1}^{\prime}-b k_{2}^{\prime} \ln \left(\frac{r}{b}\right)\right]+b k_{3}^{\prime}\left[k_{1}+a k_{2} \ln \left(\frac{r}{a}\right)\right]}{a k_{2} k_{1}^{\prime}+b k_{1} k_{2}^{\prime}+a b k_{2} k_{2}^{\prime} \ln \left(\frac{b}{a}\right)}-$

$\pi \sum_{n=1}^{\infty} \frac{e^{-D \alpha_{n}^{2} t}}{F\left(\alpha_{n}\right)} \times\left\{k_{1}^{\prime} \alpha_{n} J_{1}\left(b \alpha_{n}\right)-k_{2}^{\prime} J_{0}\left(b \alpha_{n}\right)\right\} \times$

$U_{0}\left(r \alpha_{n}\right) \times\left[\begin{array}{l}k_{3}\left\{k_{1}^{\prime} \alpha_{n} J_{1}\left(b \alpha_{n}\right)-k_{2}^{\prime} J_{0}\left(b \alpha_{n}\right)\right\}_{-} \\ k_{3}^{\prime}\left\{k_{1} \alpha_{n} J_{1}\left(a \alpha_{n}\right)+k_{2} J_{0}\left(a \alpha_{n}\right)\right\}\end{array}\right]$

where

$$
\begin{aligned}
& F\left(\alpha_{n}\right)=\left(k_{1}^{\prime 2} \alpha_{n}^{2}+k_{2}^{\prime 2}\right)\left[k_{1} \alpha_{n} J_{1}\left(a \alpha_{n}\right)+k_{2} J_{0}\left(a \alpha_{n}\right)\right]^{2}- \\
& \left(k_{1}^{2} \alpha_{n}^{2}+k_{2}^{2}\right)\left[k_{1}^{\prime} \alpha_{n} J_{1}\left(b \alpha_{n}\right)-k_{2}^{\prime} J_{0}\left(b \alpha_{n}\right)\right]^{2} \\
& U_{0}\left(r \alpha_{n}\right)=J_{0}\left(r \alpha_{n}\right)\left[k_{1} \alpha_{n} Y_{1}\left(a \alpha_{n}\right)+k_{2} Y_{0}\left(a \alpha_{n}\right)\right]- \\
& Y_{0}\left(r \alpha_{n}\right)\left[k_{1} \alpha_{n} J_{1}\left(a \alpha_{n}\right)+k_{2} J_{0}\left(a \alpha_{n}\right)\right]
\end{aligned}
$$

Here $\alpha_{n}$ are the roots of the following equation:

$\left[k_{1} \alpha J_{1}(a \alpha)+k_{2} J_{0}(a \alpha)\right]\left[k_{1}^{\prime} \alpha Y_{1}(b \alpha)-k_{2}^{\prime} Y_{0}(b \alpha)\right]-$
$\left[k_{1} \alpha Y_{1}(a \alpha)+k_{2} Y_{0}(a \alpha)\right]\left[k_{1}^{\prime} \alpha J_{1}(b \alpha)-k_{2}^{\prime} J_{0}(b \alpha)\right]=0$

(6) surfaces are

Consider the hollow cylinder with zero concentration initially and the boundary conditions on the two

$$
\begin{aligned}
& t \leq 0, a<r<b, C=0 \\
& t>0, \quad r=a, C=C_{0} \\
& t>0, \quad r=b, C=C_{0}
\end{aligned}
$$

In order to satisfy the Eq. (7), the constant of the Eq. (2) can be derived as $k_{1}=0, k_{2}=1, k_{3}=-C_{0}, k_{1}^{\prime}=0, k_{2}^{\prime}=1, k_{3}^{\prime}=C_{0}$

Substituting these constants into Eqs. (4)-(6), one obtains

$$
F\left(\alpha_{n}\right)=J_{0}^{2}\left(a \alpha_{n}\right)-J_{0}^{2}\left(b \alpha_{n}\right)
$$




$$
\begin{aligned}
& U_{0}\left(r \alpha_{n}\right)=J_{0}\left(r \alpha_{n}\right) Y_{0}\left(a \alpha_{n}\right)-Y_{0}\left(r \alpha_{n}\right) J_{0}\left(a \alpha_{n}\right) \\
& J_{0}(a \alpha) Y_{0}(b \alpha)-Y_{0}(a \alpha) J_{0}(b \alpha)=0
\end{aligned}
$$

where $\alpha_{\mathrm{n}}$ are the positive roots of Eq. (10), as given in Table 1[8, 10]. Substituting

$$
k_{1}=0, k_{2}=1, k_{3}=-C_{0}, k_{1}^{\prime}=0, k_{2}^{\prime}=1, k_{3}^{\prime}=C_{0}
$$

Eqs. (8)- (10) into Eq. (3), one obtains

$$
C=C_{0}-\pi C_{0} \sum_{n=1}^{\infty} \frac{J_{0}\left(b \alpha_{n}\right) U_{0}\left(r \alpha_{n}\right)}{J_{0}\left(a \alpha_{n}\right)+J_{0}\left(b \alpha_{n}\right)} \exp \left(-D \alpha_{n}{ }^{2} t\right)
$$

The normalized concentration of this case can be expressed as

$$
\frac{C}{C_{0}}=1-\pi \sum_{n=1}^{\infty} \frac{J_{0}\left(k a \alpha_{n}\right) U_{0}\left(a \alpha_{n} r^{*}\right)}{J_{0}\left(a \alpha_{n}\right)+J_{0}\left(k a \alpha_{n}\right)} \exp \left[-\left(a \alpha_{n}\right)^{2} \tau\right]
$$

$$
\begin{aligned}
& U_{0}\left(a \alpha_{n} r^{*}\right)=J_{0}\left(a \alpha_{n} r^{*}\right) Y_{0}\left(a \alpha_{n}\right)-J_{0}\left(a \alpha_{n}\right) Y_{0}\left(a \alpha_{n} r^{*}\right) \\
& r^{*}=\frac{r}{a}, K=\frac{b}{a}, \tau=\frac{D t}{a^{2}}
\end{aligned}
$$

\subsection{Decay transient stat}

\subsubsection{Fast decay : Case A}

Carslaw and Jaeger [9] had given the solution to the problem of diffusion into a hollow cylinder with initial concentration and the two surfaces and kept at zero concentration. The general solution is

$$
C=\frac{\pi^{2}}{2} \sum_{n=1}^{\infty} \frac{\alpha_{n}{ }^{2} J_{0}^{2}\left(a \alpha_{n}\right)}{J_{0}^{2}\left(a \alpha_{n}\right)-J_{0}^{2}\left(b \alpha_{n}\right)} \times \exp \left(-D \alpha_{n}{ }^{2} t\right) U_{0}\left(r \alpha_{n}\right) \int_{a}^{b} r f(r) U_{0}\left(r \alpha_{n}\right) d r
$$

where

$$
U_{0}\left(r \alpha_{n}\right)=J_{0}\left(r \alpha_{n}\right) Y_{0}\left(b \alpha_{n}\right)-Y_{0}\left(r \alpha_{n}\right) J_{0}\left(b \alpha_{n}\right)
$$

Here $\alpha_{n}$ are the positive roots of Eq. (10), as given in Table 1[8, 10]. Consider, Eq. (12), the concentration of the steady state, as the initial condition i.e. $\mathrm{C}=\mathrm{C}_{0}$. The boundary conditions are

$$
\begin{aligned}
& t \leq 0, a<r<b, C=C_{0} \\
& t>0, \quad r=a, C=0 \\
& t>0, \quad r=b, C=0
\end{aligned}
$$

Substituting Eqs.(14) and (15) into the integral

$$
\int_{a}^{b} r f(r) U_{0}\left(r \alpha_{n}\right) d r
$$

in Eq. (13), one obtains

$$
\int_{a}^{b} r f(r) U_{0}\left(r \alpha_{n}\right) d r=\int_{a}^{b} r C_{0} U_{0}\left(r \alpha_{n}\right) d r=C_{0} \int_{a}^{b} r U U_{0}\left(r \alpha_{n}\right) d r
$$


Since

$$
\int_{a}^{b} r U_{0}\left(r \alpha_{n}\right) d r=-\frac{1}{\alpha_{n}^{2}}\left[r \frac{d U_{0}\left(r \alpha_{n}\right)}{d r}\right]_{a}^{b}=\frac{2\left\{J_{0}\left(a \alpha_{n}\right)-J_{0}\left(b \alpha_{n}\right)\right\}}{\pi \alpha_{n}^{2} J_{0}\left(a \alpha_{n}\right)}
$$

Eq. (16) becomes

$$
\int_{a}^{b} r f(r) U_{0}\left(r \alpha_{n}\right) d r=\frac{2 C_{0}\left[J_{0}\left(a \alpha_{n}\right)-J_{0}\left(b \alpha_{n}\right)\right]}{\pi \alpha_{n}{ }^{2} J_{0}\left(a \alpha_{n}\right)}
$$

Substituting Eq. (18) into Eq. (13), one obtains

$$
C=\pi C_{0} \sum_{n=1}^{\infty} \frac{J_{0}\left(a \alpha_{n}\right) U_{0}\left(r \alpha_{n}\right)}{J_{0}\left(a \alpha_{n}\right)+J_{0}\left(b \alpha_{n}\right)} \exp \left(-D \alpha_{n}{ }^{2} t\right)
$$

Or the normalized concentration of this case is

$$
\frac{C}{C_{0}}=\pi \sum_{n=1}^{\infty} \frac{J_{0}\left(a \alpha_{n}\right) U_{0}\left(a \alpha_{n} r_{A}^{*}\right)}{J_{0}\left(a \alpha_{n}\right)+J_{0}\left(k a \alpha_{n}\right)} \exp \left[-\left(a \alpha_{n}\right)^{2} \tau\right]
$$

$$
\begin{aligned}
& U_{0}\left(a \alpha_{n} r_{A}^{*}\right)=J_{0}\left(a \alpha_{n} r_{A}^{*}\right) Y_{0}\left(k a \alpha_{n}\right)-Y_{0}\left(a \alpha_{n} r_{A}^{*}\right) J_{0}\left(k a \alpha_{n}\right) \\
& r_{A}^{*}=\frac{r}{a} \quad K=\frac{b}{a} \quad \tau=\frac{D t}{a^{2}}
\end{aligned}
$$

Table 1

Roots of $J_{0}\left(a \alpha_{n}\right) Y_{0}\left(b \alpha_{n}\right)-J_{0}\left(b \alpha_{n}\right) Y_{0}\left(a \alpha_{n}\right)$.

\begin{tabular}{|cccccc|}
\hline$\frac{b}{a}$ & $a \alpha_{1}$ & $a \alpha_{2}$ & $a \alpha_{3}$ & $a \alpha_{4}$ & $a \alpha_{5}$ \\
\hline 1.2 & 15.7014 & 31.4126 & 47.1217 & 62.8302 & 78.5385 \\
1.5 & 6.2702 & 12.5598 & 18.8451 & 25.1294 & 31.4133 \\
2.0 & 3.1230 & 6.2734 & 9.4182 & 12.5614 & 15.7040 \\
2.5 & 2.0732 & 4.1773 & 6.2754 & 8.3717 & 10.4672 \\
3.0 & 1.5485 & 3.1291 & 4.7038 & 6.2767 & 7.8487 \\
3.5 & 1.2339 & 2.5002 & 3.7608 & 5.0196 & 6.2776 \\
4.0 & 1.0244 & 2.0809 & 3.1322 & 4.1816 & 5.2301 \\
\hline
\end{tabular}

\subsubsection{Slow decay: Case B}

Özişik [12] had given the solution to the problem of diffusion into a hollow cylinder by the method of separation of variables with initial concentration $F(r)$, the two surfaces $\mathrm{r}=$ a with zero flux and kept at zero concentration. The general solution is

$$
C=\sum_{n=1}^{\infty} \frac{1}{N\left(\alpha_{n}\right)} e^{-D \alpha_{n}{ }^{2} t} R_{0}\left(\alpha_{n} r\right) \int_{a}^{b} r R_{0}\left(\alpha_{n} r\right) F(r) d r
$$


Where

$$
\begin{aligned}
& R_{0}\left(\alpha_{n} r\right)=J_{0}\left(\alpha_{n} r\right) Y_{0}\left(\alpha_{n} b\right)-J_{0}\left(\alpha_{n} b\right) Y_{0}\left(\alpha_{n} r\right) \\
& \frac{1}{N\left(\alpha_{n}\right)}=\frac{\pi^{2}}{2} \times \frac{\alpha_{n}^{2} J_{1}^{2}\left(\alpha_{n} a\right)}{J_{1}^{2}\left(\alpha_{n} a\right)-J_{0}^{2}\left(\alpha_{n} b\right)}
\end{aligned}
$$

And

$$
J_{1}(a \alpha) Y_{0}(b \alpha)-J_{0}(b \alpha) Y_{1}(a \alpha)=0
$$

Here $\alpha_{n}$ are the positive roots of Eq. (25) which were determined as given in Table 2.

Table 2

Roots of $J_{1}\left(a \alpha_{n}\right) Y_{0}\left(b \alpha_{n}\right)-J_{0}\left(b \alpha_{n}\right) Y_{1}\left(a \alpha_{n}\right)$.

\begin{tabular}{|cccccc|}
\hline$\frac{b}{a}$ & $a \alpha_{1}$ & $a \alpha_{2}$ & $a \alpha_{3}$ & $a \alpha_{4}$ & $a \alpha_{5}$ \\
\hline 1.2 & 8.14628 & 23.661 & 39.3308 & 55.0214 & 70.7197 \\
1.5 & 3.4029 & 9.52064 & 15.766 & 22.0327 & 28.3067 \\
2.0 & 1.79401 & 4.80206 & 7.90896 & 11.0351 & 14.168 \\
2.5 & 1.24267 & 3.22655 & 5.28885 & 7.36856 & 9.45462 \\
3.0 & 0.959569 & 2.43717 & 3.97818 & 5.53497 & 7.09771 \\
3.5 & 0.78554 & 1.96238 & 3.19134 & 4.43458 & 5.68343 \\
4.0 & 0.666971 & 1.645 & 2.66644 & 3.70083 & 4.74048 \\
\hline
\end{tabular}

In this case, consider, Eq.(12), the concentration of the steady state, as the initial condition i.e. $\mathrm{C}=\mathrm{C}_{0}$. The boundary conditions are

$$
\begin{aligned}
& t \leq 0, a<r<b, C=C_{0} \\
& t>0, \quad r=a, \frac{\partial C}{\partial r}=0 \\
& t>0, \quad r=b, C=0
\end{aligned}
$$

Substituting Eq. (23) and (26) into the integral

$$
\int_{a}^{b} r R_{0}\left(r \alpha_{n}\right) F(r) d r
$$

$a \quad$ in Eq. (22), one obtains

$$
\begin{aligned}
& \int_{a}^{b} r R_{0}\left(r \alpha_{n}\right) F(r) d r=C_{0} \int_{a}^{b} r\left\{J_{0}\left(\alpha_{n} r\right) Y_{0}\left(\alpha_{n} b\right)-J_{0}\left(\alpha_{n} b\right) Y_{0}\left(\alpha_{n} r\right)\right\} d r \\
& C_{0}\left\{\frac{2-a \pi \alpha_{n}\left[J_{1}\left(\alpha_{n} a\right) Y_{0}\left(\alpha_{n} b\right)-J_{0}\left(\alpha_{n} b\right) Y_{1}\left(\alpha_{n} a\right)\right]}{\pi \alpha_{n}^{2}}\right\}
\end{aligned}
$$

Substituting Eq. (27) into Eq. (22), one obtains

$$
\begin{aligned}
& C=\sum_{n=1}^{\infty} \frac{\pi C_{0}}{2} \times \frac{\left\{2-a \pi \alpha_{n}\left[J_{1}\left(\alpha_{n} a\right) Y_{0}\left(\alpha_{n} b\right)-J_{0}\left(\alpha_{n} b\right) Y_{1}\left(\alpha_{n} a\right)\right]\right\} J_{1}^{2}\left(\alpha_{n} a\right) R_{0}\left(\alpha_{n} r\right)}{J_{1}^{2}\left(\alpha_{n} a\right)-J_{0}^{2}\left(\alpha_{n} b\right)} \\
& \times \exp \left(-D \alpha_{n}{ }^{2} t\right)
\end{aligned}
$$


The normalized concentration of this case is

$$
\begin{aligned}
& \frac{C}{C_{0}}=\sum_{n=1}^{\infty} \frac{\pi}{2} \times \frac{\left\{2-\pi \alpha_{n} a\left[J_{1}\left(\alpha_{n} a\right) Y_{0}\left(k \alpha_{n} a\right)-J_{0}\left(k \alpha_{n} a\right) Y_{1}\left(\alpha_{n} a\right)\right]\right\} J_{1}^{2}\left(\alpha_{n} a\right) R_{0}\left(\alpha_{n} a r_{B}^{*}\right)}{J_{1}^{2}\left(\alpha_{n} a\right)-J_{0}^{2}\left(k \alpha_{n} a\right)} \\
& \times \exp \left(-\alpha_{n}{ }^{2} a^{2} \tau\right)
\end{aligned}
$$

where

$$
\begin{aligned}
& R_{0}\left(a \alpha_{n} r_{B}^{*}\right)=J_{0}\left(a \alpha_{n} r_{B}^{*}\right) Y_{0}\left(k a \alpha_{n}\right)-J_{0}\left(k a \alpha_{n}\right) Y_{0}\left(a \alpha_{n} r_{B}^{*}\right) \\
& r_{B}^{*}=\frac{r}{a} \quad K=\frac{b}{a} \quad \tau=\frac{D t}{a^{2}}
\end{aligned}
$$

\subsubsection{Slow decay: Case C}

Özişik [12] had given the solution to the problem of diffusion into a hollow cylinder by the method of separation of variables with initial concentration $F(r)$, the two surfaces kept at zero concentration and $\mathrm{r}=\mathrm{a}$ with zero flux. The general solution is

$$
C=\sum_{n=1}^{\infty} \frac{1}{N\left(\alpha_{n}\right)} e^{-D \alpha_{n}{ }^{2} t} R_{0}\left(\alpha_{n} r\right) \int_{a}^{b} r R_{0}\left(\alpha_{n} r\right) F(r) d r
$$

where

$$
\begin{aligned}
& R_{\mathrm{O}}\left(\alpha_{n} r\right)=J_{1}\left(\alpha_{n} b\right) Y_{\mathrm{O}}\left(\alpha_{n} r\right)-J_{\mathrm{O}}\left(\alpha_{n} r\right) Y_{1}\left(\alpha_{n} b\right) \\
& \frac{1}{N\left(\alpha_{n}\right)}=\frac{\pi^{2}}{2} \times \frac{\alpha_{n}^{2} J_{\mathrm{O}}^{2}\left(\alpha_{n} a\right)}{J_{0}^{2}\left(\alpha_{n} a\right)-J_{1}^{2}\left(\alpha_{n} b\right)} \\
& J_{\mathrm{O}}(a \alpha) Y_{1}(b \alpha)-J_{1}(b \alpha) Y_{\mathrm{O}}(a \alpha)=0
\end{aligned}
$$

Here $\alpha_{n}$ are the positive roots of Eq. (34) which were determined as given in Table 3.

Table 3

Roots of $J_{0}\left(a \alpha_{n}\right) Y_{1}\left(b \alpha_{n}\right)-J_{1}\left(b \alpha_{n}\right) Y_{0}\left(a \alpha_{n}\right)$.

\begin{tabular}{|cccccc|}
\hline$\frac{b}{a}$ & $a \alpha_{1}$ & $a \alpha_{2}$ & $a \alpha_{3}$ & $a \alpha_{4}$ & $a \alpha_{5}$ \\
1.2 & 7.56667 & 23.4688 & 39.2141 & 54.9381 & 70.6549 \\
1.5 & 2.88989 & 9.34479 & 15.6601 & 21.957 & 28.2478 \\
2.0 & 1.36078 & 4.6459 & 7.81416 & 10.9671 & 14.1151 \\
2.5 & 0.866058 & 3.08354 & 5.20107 & 7.30541 & 9.40534 \\
3.0 & 0.625598 & 2.30404 & 3.89542 & 5.47516 & 7.05095 \\
3.5 & 0.485007 & 1.83725 & 3.11249 & 4.37731 & 5.63854 \\
4.0 & 0.393456 & 1.52661 & 2.59082 & 3.64558 & 4.69706 \\
\hline
\end{tabular}

In this case, consider the concentration of the steady state ( $\mathrm{C}=\mathrm{Co}$ ), as the initial condition. The boundary conditions are

$$
\begin{aligned}
& t \leq 0, a<r<b, C=C_{0} \\
& t>0, \quad r=a, C=0 \\
& t>0, \quad r=b, \frac{\partial C}{\partial r}=0
\end{aligned}
$$


Substituting Eqs. (32) and (35) into the integral

$b$

$$
\int r R_{0}\left(r \alpha_{n}\right) F(r) d r
$$

a

in Eq. (31), one obtains

$$
\begin{aligned}
& \int_{a}^{b} r R_{0}\left(r \alpha_{n}\right) F(r) d r=C_{0} \int_{a}^{b} r\left\{J_{1}\left(\alpha_{n} b\right) Y_{0}\left(\alpha_{n} r\right)-J_{0}\left(\alpha_{n} r\right) Y_{1}\left(\alpha_{n} b\right)\right\} d r \\
& C_{0}\left\{\frac{a\left[J_{1}\left(\alpha_{n} a\right) Y_{1}\left(\alpha_{n} b\right)-J_{1}\left(\alpha_{n} b\right) Y_{1}\left(\alpha_{n} a\right)\right]}{\alpha_{n}}\right\}
\end{aligned}
$$

Substituting Eq. (36) into Eq. (31), one obtains

$$
\begin{aligned}
& C=\sum_{n=1}^{\infty} \frac{\pi^{2} C_{0}}{2} \times \frac{\left.\alpha_{n} a J_{0}^{2}\left(\alpha_{n} a\right)\left[J_{1}\left(\alpha_{n} a\right) Y_{1}\left(\alpha_{n} b\right)-J_{1}\left(\alpha_{n} b\right) Y_{1}\left(\alpha_{n} a\right)\right]\right\} R_{0}\left(\alpha_{n} r\right)}{J_{0}^{2}\left(\alpha_{n} a\right)-J_{1}^{2}\left(\alpha_{n} b\right)} \\
& \times \exp \left(-D \alpha_{n}^{2} t\right)
\end{aligned}
$$

The normalized concentration of this case is

$$
\begin{aligned}
& \frac{C}{C_{0}}=\sum_{n=1}^{\infty} \frac{\pi^{2}}{2} \times \frac{\left.\alpha_{n} a J_{0}^{2}\left(\alpha_{n} a\right)\left[J_{1}\left(\alpha_{n} a\right) Y_{1}\left(k \alpha_{n} a\right)-J_{1}\left(k \alpha_{n} a\right) Y_{1}\left(\alpha_{n} a\right)\right]\right\} R_{0}\left(\alpha_{n} a r_{C}^{*}\right)}{J_{0}^{2}\left(\alpha_{n} a\right)-J_{1}^{2}\left(k \alpha_{n} a\right)} \\
& \times \exp \left(-\alpha_{n}^{2} a^{2} \tau\right) \\
& R_{0}\left(a \alpha_{n} r_{C}^{*}\right)=J_{1}\left(k a \alpha_{n}\right) Y_{0}\left(a \alpha_{n} r_{C}^{*}\right)-J_{0}\left(a \alpha_{n} r_{C}^{*}\right) Y_{1}\left(k a \alpha_{n}\right) \\
& r_{C}^{*}=\frac{r}{a} \quad K=\frac{b}{a} \quad \tau=\frac{D t}{a^{2}}
\end{aligned}
$$

\section{RESULTS AND DISCUSSION}

\subsection{Set up transient state}

The concentration distribution of $\mathrm{C} / \mathrm{C}_{\mathrm{O}}$ predicted by Eq. (12) are plotted against $\mathrm{r}^{*}$ for $\mathrm{K}=1.2,1.5,2$, 2.5, 3, 3.5 and 4, at, 0.4 and 1 as shown in Figs. 1(a)-(c), respectively. These curves are obtained by using the first five roots, $\alpha_{n}$ 's, of the Eq. (10) which are listed in Table 1.The concentration profile increases with increasing $\tau$ and decreasing K. Generally, the concentration profiles of this case are symmetrical. All curves reveal similar concave shapes. For $\mathrm{K}=1.2$ and 1.5, there is no difference between Figs. 1(a) and (b) since steady state has already been reached at $\tau \geqq 0.2$. Also, these curves indicate that the greater the value of $K$, the more $\tau$ is needed to reach a steady state.

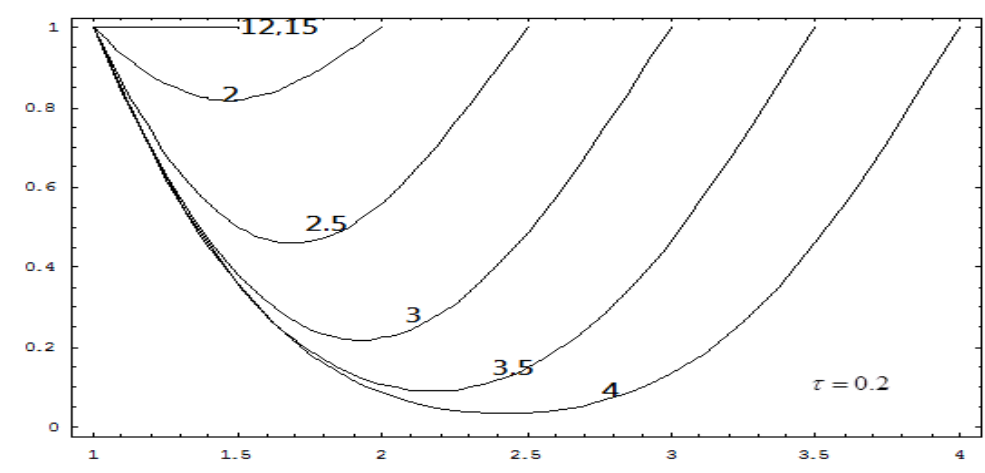

Fig.1(a) 


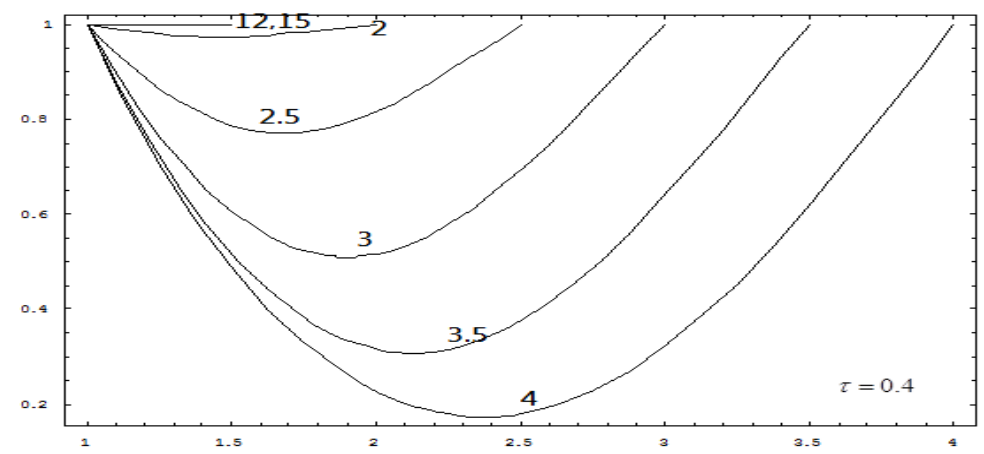

Fig.1(b)

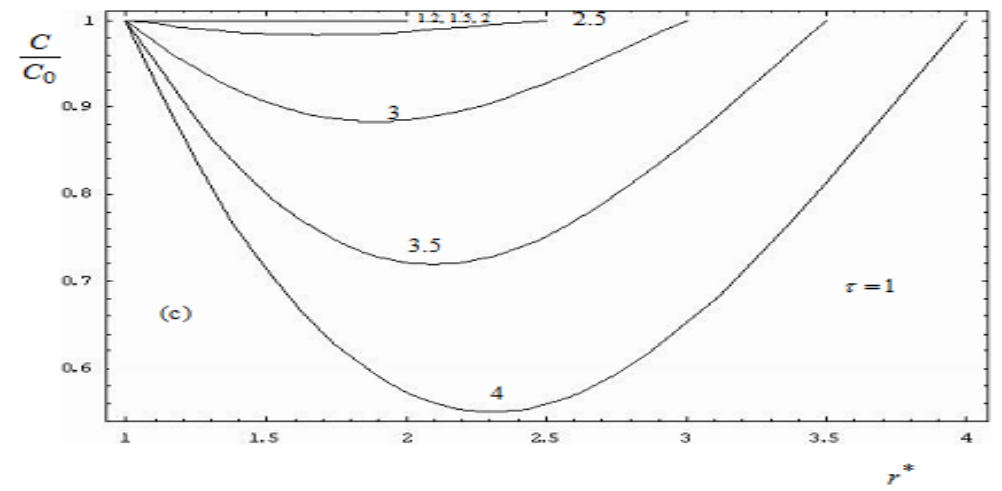

Fig.1(c)

Fig. 1. Set up transient state concentration distribution through cylinder wall at (a) $\tau=0.2$ (b) $\tau=0.4$ (c) $\tau=1$. Numbers on curves are values of K.

\subsection{Decay transient state}

\subsubsection{Fast decay: Case $A$}

The concentration distribution of $C / C_{O}$ calculated by Eq. (20) are plotted against $r_{A}{ }^{*}$ at various $\tau$ and K, as shown in Figs. 2(a)-(c), respectively. These curves are also plotted by using the first five roots, $\alpha_{\mathrm{n}}$ 's, of the Eq. (10) which are listed in Table 1. The concentration profile decreases with increasing $\succ$ and decreasing $\mathrm{K}$. The concentration profiles are symmetrical. Especially for $\mathrm{K} \leqq 1.2$, it is almost as symmetrical as the sheet case [13], as shown in Fig. 2(a). All curves reveal similar convex shapes.

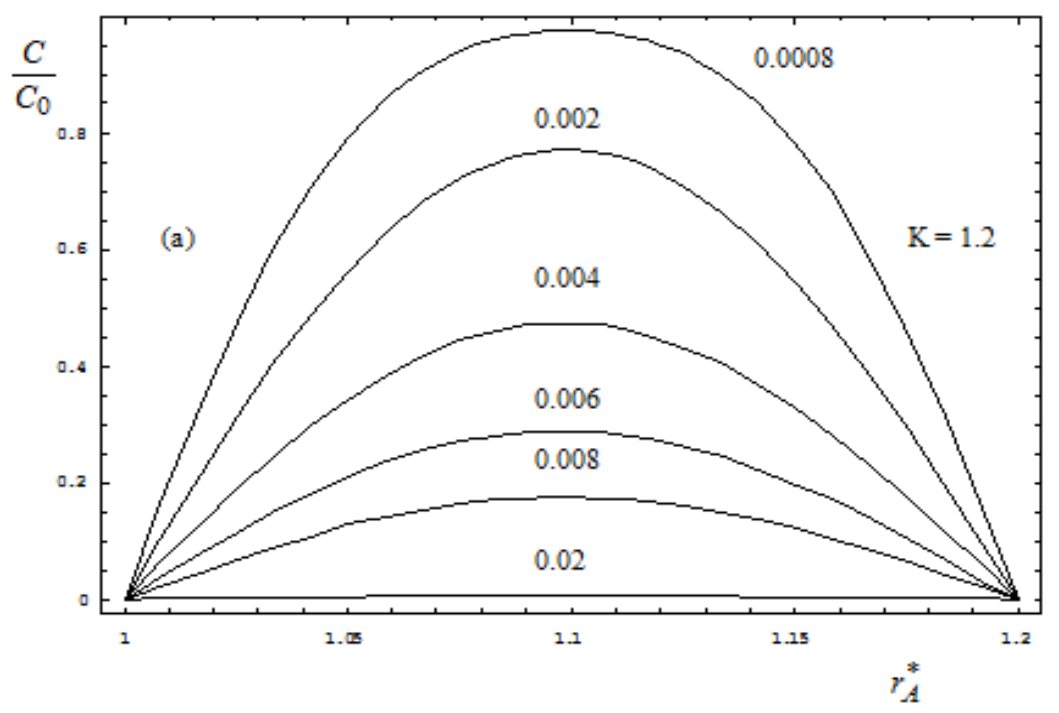

Fig.2(a) 


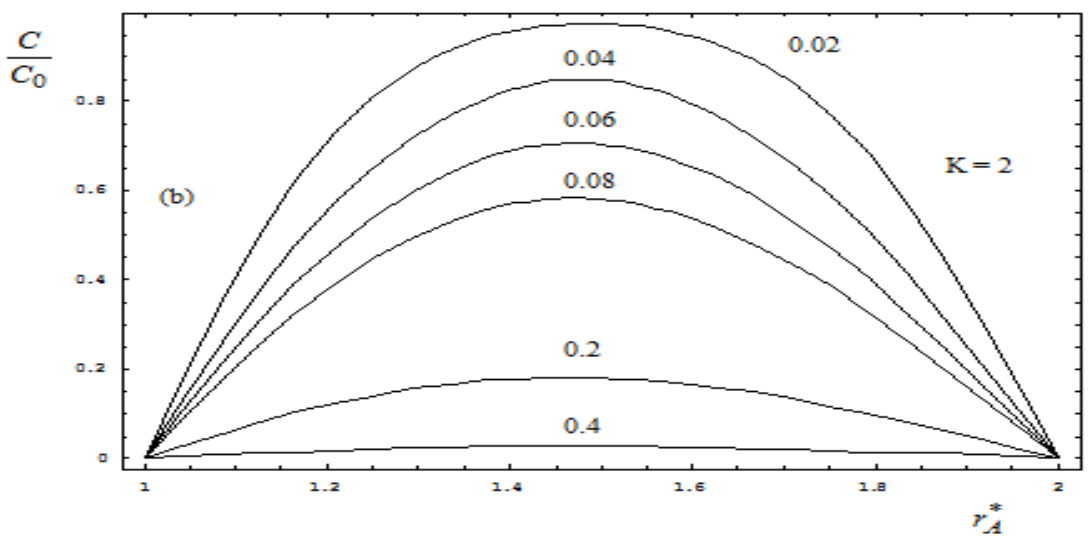

Fig.2(b)

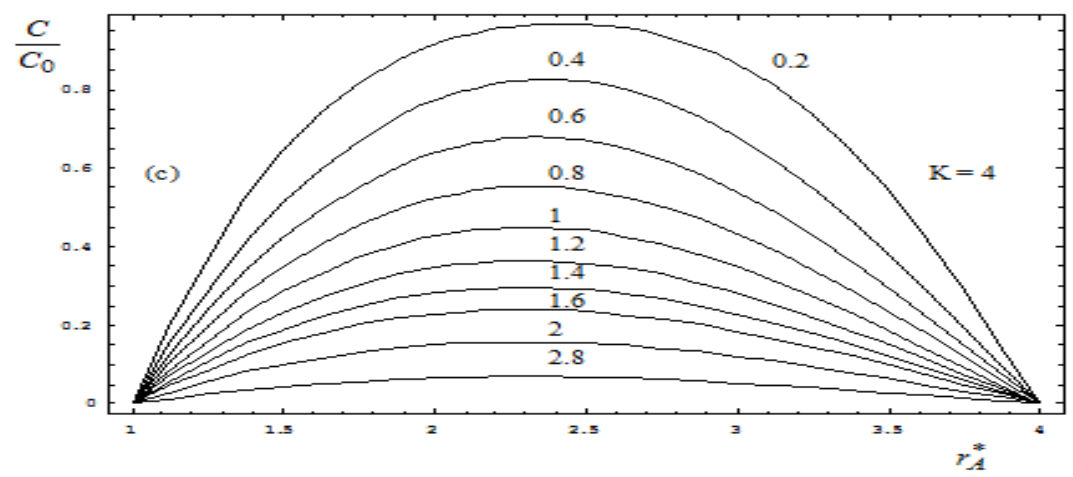

Fig.2(c)

Fig. 2. Decay transient state concentration distribution through cylinder wall for (a) $K=1.2$ (b) $K=2$ (c) $\mathrm{K}=4$. Numbers on curves are values of for case $\mathrm{A}$.

\subsubsection{Slow decay: Case B}

The concentration distribution of $\mathrm{C} / \mathrm{C}_{\mathrm{O}}$ calculated by Eq. (29) are plotted against $\mathrm{r}_{\mathrm{B}}{ }^{*}$ at various $\tau$ and $\mathrm{K}$, as shown in Figs. 3(a)-(c), respectively. These curves are also plotted by using the first five roots, $\alpha_{\mathrm{n}}$ 's, of the Eq. (25) which are listed in Table 2. The concentration profile decreases with increasing $\tau$ and decreasing $\mathrm{K}$. All curves reveal similar convex shapes.

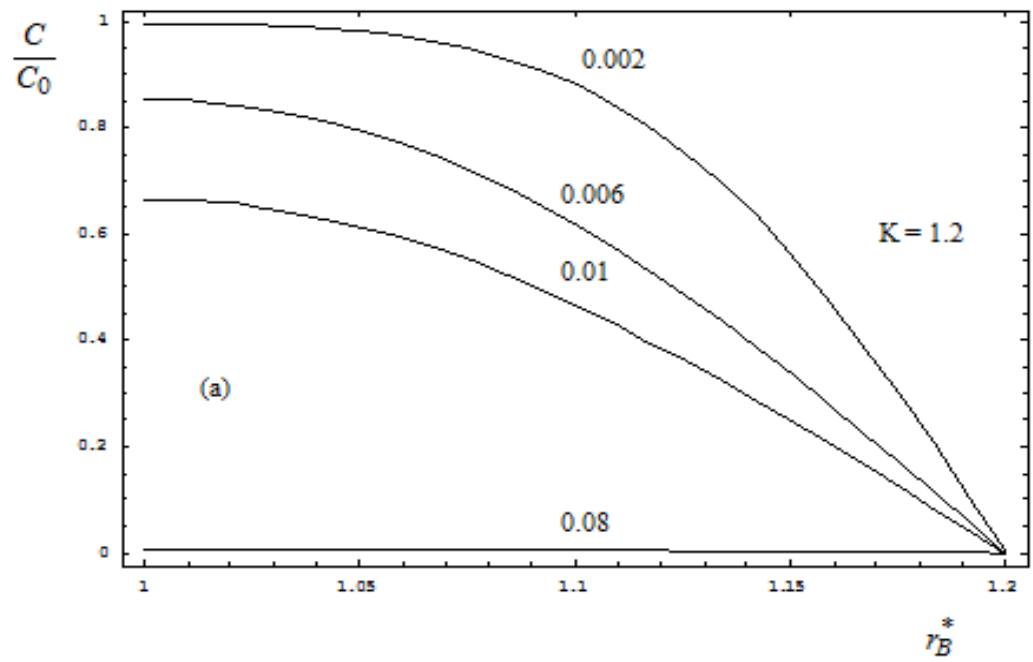

Fig.3(a) 


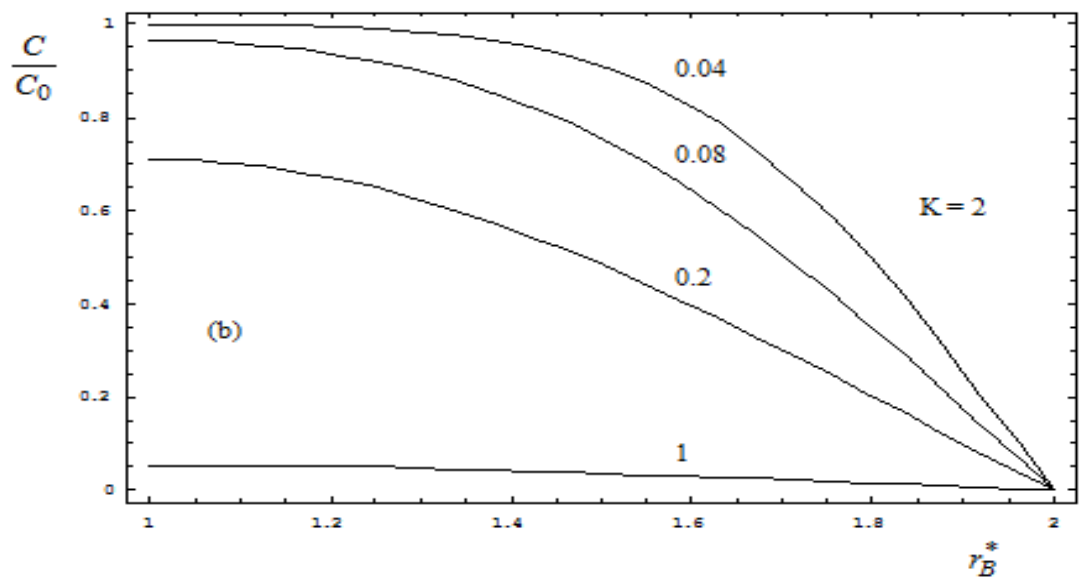

Fig.3(b)

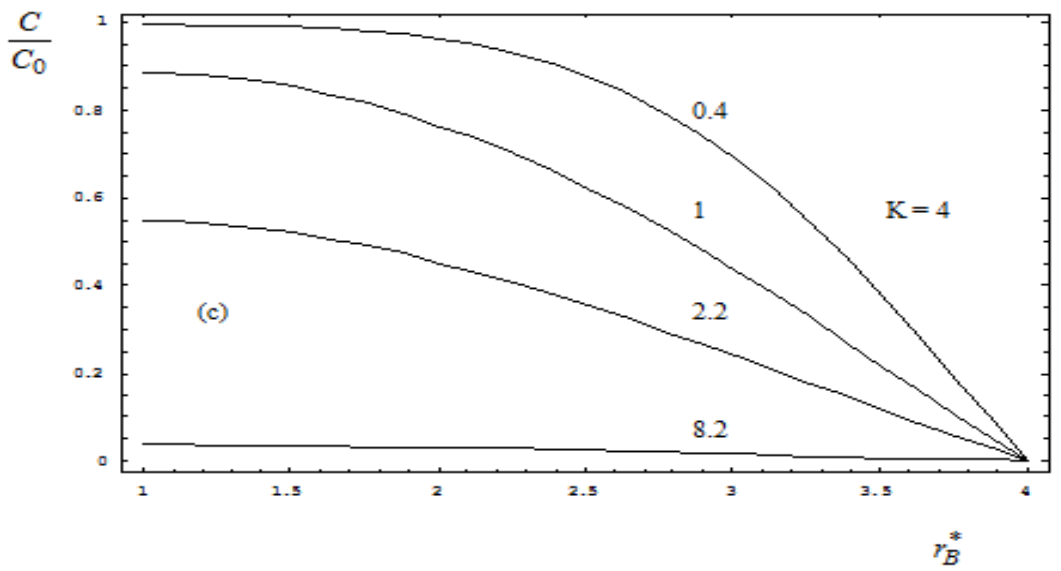

Fig.3(c)

Fig. 3. Decay transient state concentration distribution through cylinder wall for (a) $K=1.2(b) K=2$ (c) $\mathrm{K}=4$. Numbers on curves are values of for case $\mathrm{B}$.

\subsubsection{Slow decay: Case C}

The concentration distribution of $C / C_{O}$ calculated by Eq. (38) are plotted against $r_{c}{ }^{*}$ at various $\tau$ and K, as shown in Figs. 4(a)-(c), respectively. These curves are also plotted by using the first five roots, $\alpha_{\mathrm{n}}$ 's, of the Eq. (34) which are listed in Table 3. The concentration profile decreases with increasing $\tau$ and decreasing $\mathrm{K}$. All curves reveal similar convex shapes. Comparing the decreasing rate of concentration profile for cases A, B and $\mathrm{C}$, case $\mathrm{A}$ is the fastest, case $\mathrm{C}$ is the slowest for $\mathrm{K} \geqq 2$. However, for $\mathrm{K} \leqq 1.2$, the decreasing rate of concentration profile for cases $\mathrm{B}$ and $\mathrm{C}$ is the same.

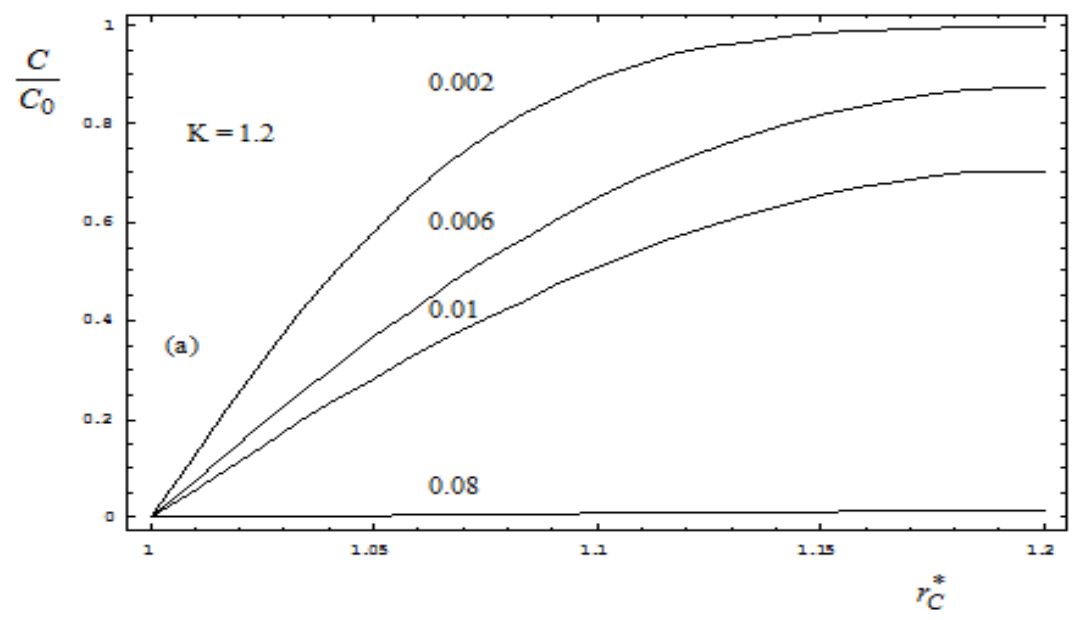

Fig.4 (a) 


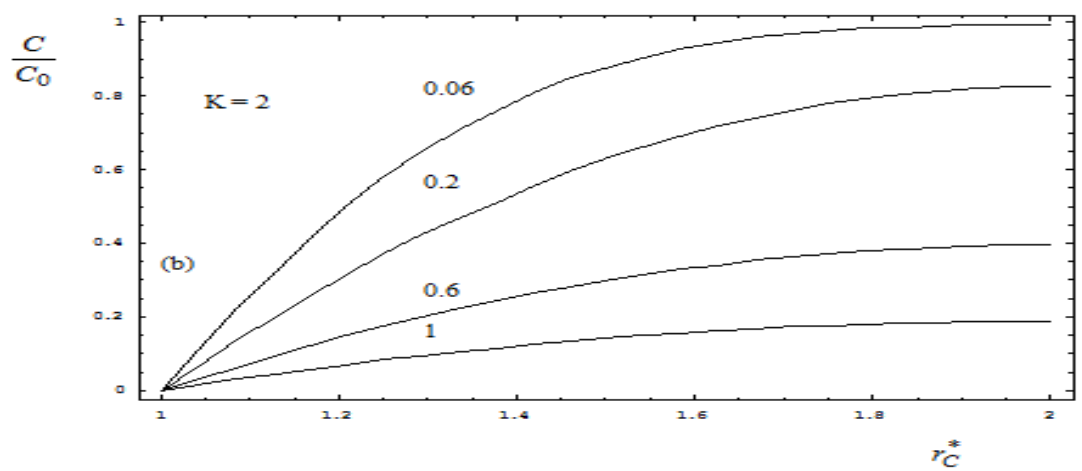

Fig.4 (b)

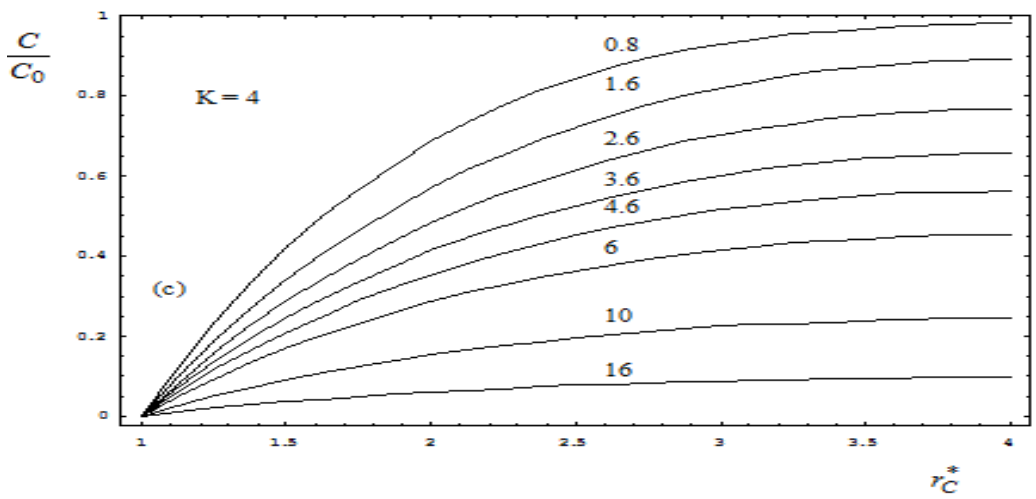

Fig.4(c)

Fig. 4. Decay transient state concentration distribution through cylinder wall for (a) $\mathrm{K}=1.2$ (b) $\mathrm{K}=2$ (c) $\mathrm{K}=4$. Numbers on curves are values of for case $\mathrm{C}$.

\section{CONCLUSION}

The mathematical solutions of diffusion in hollow cylinder with different $\mathrm{K}$ for the set up transient and decay transient states are given in Eqs. (12), (20), (29), and (38), respectively. Through the mathematical analysis and figure plotting, a few conclusions are drawn:

1. In the set up transient state and decay transient state for case A, the concentration profiles are reverse and symmetrical.

2. In the decay transient state for cases B and C, the normalized concentration profiles are reverse and reveal the similar convex shapes.

3. In decay transient states for cases $\mathrm{A}, \mathrm{B}$ and $\mathrm{C}$, comparing the decreasing rate of the concentration profile, case $\mathrm{A}$ is the fastest, for the same $\mathrm{K}$.

\section{REFERENCES}

[1] M. A. V Devanathan and Z. Stachurski, The adsorption and diffusion of electrolytic hydrogen in palladium, Proceedings of the Royal Society, A 270, 1962, 90-102.

[2] J. McBreen, L. Nanis, and W. Beck, A Method for Determination of the Permeation Rate of Hydrogen Through Metal Membranes, Journal of the Electrochemical Society, 113,1966, 1218-1222.

[3] K. Kiuchi and R. B. McLellan, The Solubility and Diffusivity of Hydrogen in Well-Annealed and Deformed Iron, Acta Metallurgica 31,1983, 961-984.

[4] S. K. Yen and H. C. Shih, A Mathematical Solution to the Determination of the Permeation Rate of Hydrogen through Metal Membrane, Journal of the Electrochemical Society, 135,1988, 1169-1170.

[5] R. W. Revie, V. S. Sastri, G. R. Hoey, R. R. Ramsingh, D. K. Mak, and M. T. Shehata, Hydrogen-Induced Cracking of Linepipe Steels Part 1-Threshold Hydrogen Concentration and pH, Corrosion, 49 1993,17-23.

[6] R. W. Revie, V. S. Sastri, M. Elboujdaini, R. R. Ramsingh, and Y. Lafreni re, Hydrogen-Induced Cracking of Line Pipe Steels Used in Sour Service, Corrosion, 49,1993, 531-535.

[7] R. Ash, R. M. Barrer, D. G. Palmer, Diffusion in multiple laminates, British Journal of Applied Physics 16 ,1965, 873-884

[8] H. S. Carslaw and J. C. Jaeger, Conduction of Heat in Solids, 2nd Edition, Oxford, 1959, 332-333.

[9] H. S. Carslaw and J. C. Jaeger, Conduction of Heat in Solids, 2nd Edition, Oxford, 1959, 205-207.

[10] J. Crank, The Mathematics of Diffusion, Oxford University Press, Oxford, 1975, chapter 5.

[11] I. B. Huang and S. K. Yen, Diffusion in hollow cylinders for some boundary conditions I. Mathematical treatment, Materials Chemistry and Physics $74,2002,289-299$.

[12] M. Necati Özișik, Heat Conduction, 2nd Edition, New York, 1993, 112-113.

[13] S. K. Yen and Y. C. Tsai, Critical Hydrogen Concentration For The Brittle Fracture of AISI 430 Stainless Steel, Journal of the Electrochemical Society, 143,1996, 2736-2741. 